\title{
Uso de nitazoxanida en infecciones virales
}

\author{
Use of nitazoxanide in viral infections
}

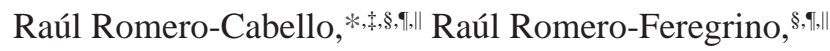

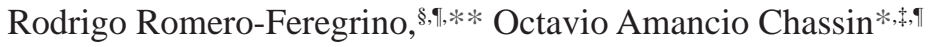 \\ * Hospital General de México «Dr. Eduardo Liceaga». México. \\ ₹ Facultad de Medicina de la Universidad Nacional Autónoma de México. México. \\ $\S$ Instituto para el Desarrollo Integral de la Salud. México. \\ " Saint Luke, Escuela de Medicina. México. \\ " Academia Mexicana de Pediatría. México. \\ ** Instituto Mexicano del Seguro Social. México.
}

\section{RESUMEN}

A partir del 2019, se demostró que la nitazoxanida inhibe la replicación del virus Ébola y de la estomatitis vesicular, amplifica la respuesta antiviral del huésped, suprime la producción de citocinas proinflamatorias en células mononucleares, inhibe síntesis de interleucina 6 (IL-6), estimula sistema inmune innato y atenúa la respuesta inmunológica e inflamatoria. Tiene acción contra MERS-CoV y otros coronavirus, tiene actividad antiviral contra virus respiratorios influenza $A$ y $B$ y parainfluenza. La tizoxanida alcanza concentraciones inhibitorias efectivas para producir su efecto antiviral en los diferentes órganos y actividad in vitro contra SARS-CoV-2, mediante amplificación de respuesta antiviral del interferón, incrementa la RIG-1 (sensor RNA citoplasmático), GADD34 (proteína induce daño DNA y detiene crecimiento), PKR (proteíncinasa R) y efectores MAVS, interferones y múltiples ISG (genes estimulador interferón), además de inhibir producción de citocinas proinflamatorias. Se observó con nitazoxanida disminución de carga viral de SAR-CoV-2 y mejoría de manifestaciones clínicas en casos de COVID-19.

Palabras clave: Nitazoxanida, COVID-19, SARS-CoV-2, infecciones virales, SARS-CoV, coronavirus.

\section{INTRODUCCIÓN}

La nitazoxanida nació como un antiparasitario de amplio espectro y con el tiempo se ha identificado que tiene actividad contra microorganismos de tipo bacteriano y viral; de este último grupo, se han realizado estudios de investigación que demuestran

Citar como: Romero-Cabello R, Romero-Feregrino R, Romero-Feregrino R, Amancio CO. Uso de nitazoxanida en infecciones virales. Rev Latin Infect Pediatr. 2021; 34 (2): 94-99. https://dx.doi.org/10.35366/100548

Recibido: 16-06-2021. Aceptado: 20-06-2021.

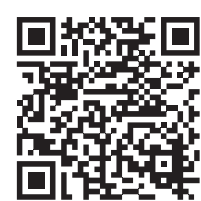

ABSTRACT

Nitazoxanide as of 2019 was shown to inhibit replication of Ebola virus and vesicular stomatitis, amplify host antiviral response, suppresses the production of proinflammatory cytokines in mononuclear cells, inhibits interleukin 6 (IL-6) synthesis, stimulates the innate immune system and attenuates the immune and inflammatory response. It has action against MERS-CoV and other coronaviruses, has antiviral activity against respiratory viruses influenza $A$ and $B$ and parainfluenza. Tizoxanide reaches effective inhibitory concentrations to produce its antiviral effect in different organs and in vitro activity against SARS-CoV-2, by amplification of antiviral interferon response, increases RIG-1 (cytoplasmic RNA sensor), GADD34 (protein induces DNA damage and growth arrest), PKR (protein kinase $R$ ) and effectors MAVS, interferons and multiple ISGs (interferon stimulator genes). In addition to inhibiting the production of proinflammatory cytokines. A decrease in SAR-CoV-2 viral load and improvement of clinical manifestations in cases of COVID-19 was observed with nitazoxanide.

Keywords: Nitazoxanide, COVID-19, SARS-CoV-2, viral infections, SARS-CoV, coronavirus.

su utilidad como medicamento antiviral contra el virus de la hepatitis $B$ y $C$, para virus de localización gastrointestinal y virus respiratorios, en particular virus de la influenza y algunos coronavirus. ${ }^{1}$

En el momento histórico que vivimos con la pandemia de COVID-19, y ante la urgencia de contar con medidas terapéuticas, se están realizando diversos trabajos de investigación para prueba de diferentes fármacos. De este grupo de medicamentos, surge la nitazoxanida como una posibilidad a explorar para enfrentar la infección por SARS-CoV-2, entre los reportes de uso empírico para tratamiento de COVID-19 se ha propuesto la administración de 
Rev Latin Infect Pediatr. 2021; 34 (2): 94-99

nitazoxanida cada 12 horas a los contactos de casos con COVID-19 y a los pacientes con enfermedad de leve a moderada.

La nitazoxanida es un tiazólido antiinfeccioso de amplio espectro autorizado en 2004 por la Food and Drug Administration (FDA) de Estados Unidos de América (EUA) para el tratamiento de parasitosis por protozoarios y helmintos. ${ }^{1,2}$ Sin embargo, su potencia contra las especies A y B de los virus Orthomyxoviridae, productores de influenza, quedó fehacientemente documentada en 2009, y después se confirmó en estudios clínicos controlados de fases II y III publicados entre 2014 y $2017 . .^{1,2}$

\section{ACCIÓN ANTIVIRAL}

A la luz de la evidencia recabada en los últimos cinco años, la nitazoxanida se ha recomendado y utilizado en todo el mundo para el tratamiento de infecciones virales respiratorias, gastrointestinales y por virus de la hepatitis de los tipos $\mathrm{B}$ y $\mathrm{C}(\mathrm{VHB}, \mathrm{VHC})$ en más de 75 millones de pacientes, pediátricos y adultos. ${ }^{1,2}$ Adicionalmente, en el transcurso de la más reciente década, se ha hecho evidente una importante actividad antibacteriana por parte de la tizoxanida (el metabolito circulante de la nitazoxanida) contra un amplio espectro de anaerobios obligados y facultativos, tanto grampositivos como gramnegativos. ${ }^{2}$

En 2019, los estudios mostraron que la nitazoxanida dispone de una potente capacidad para inhibir la replicación de los virus del Ébola (VE) y de la estomatitis vesicular (VEV), además de que amplifica la respuesta antiviral del huésped al potenciar las vías de los interferones de tipo I (IFN-I) ante la exposición al ácido ribonucleico (ARN) citoplasmático exógeno. ${ }^{3}$

Por último, los resultados de análisis in vitro y los experimentos preclínicos efectuados desde 2016 hasta la fecha han revelado que la nitazoxanida no sólo suprime la producción de citocinas proinflamatorias en las células mononucleares de la sangre periférica, sino que también inhibe la síntesis de interleucina de tipo 6 (IL-6). ${ }^{4}$ La nitazoxanida produce estimulación del sistema inmune innato y atenuación de la respuesta inmunológica e inflamatoria. ${ }^{5}$

Estos últimos efectos se asocian en forma directa con una destacada actividad contra el coronavirus productor del síndrome respiratorio de Oriente Medio (MERS-CoV: Middle East Respiratory Syndrome Coronavirus) y otros coronavirus: estos hechos ubican a la nitazoxanida como uno de los fármacos más promisorios en el combate contra los microorga- nismos patógenos que atentan contra la salud pública en el contexto mundial de nuestros días. ${ }^{4}$

\section{NITAZOXANIDA. ESTRUCTURA QUÍMICA Y MECANISMO DE ACCIÓN}

La nitazoxanida se ubica en el grupo de los tiazólidos (nitrotiazólidos); a diferencia de los nitroimidazoles, dispone de mayor espectro, mayor potencia y menor toxicidad; cuenta con cuatro distintos mecanismos de acción, dependiendo del patógeno al que esté dirigida: 6,7

1. Al actuar contra bacterias (principalmente anaerobias), interrumpe el metabolismo (energético) de estos patógenos mediante la inhibición del ciclo ferredoxina-flavodoxina-oxidorreductasa.

2. Al actuar contra protozoarios, inhibe las enzimas quinona-oxidorreductasa, nitrorreductasa 1 y proteína disulfuro-isomerasa para lesionar la membrana celular del microorganismo y despolarizar su membrana mitocondrial.

3. Al actuar contra nemátodos, inhibe la glutatióntransferasa y modula el gen Avr-14 que codifica para la subunidad $\alpha$ del canal iónico cloruroglutamato.

4. Al actuar contra virus, suprime la replicación mediante el bloqueo de la maduración de la hemaglutinina viral y la inhibición del factor de transcripción viral, además de que activa el factor de iniciación de la traducción eucariota de tipo 2- $\alpha$ (una proteína intracelular antiviral).

\section{INDICACIONES TERAPÉUTICAS}

Dado el cuádruple mecanismo de acción del que consta la nitazoxanida, este tiazólido está indicado para el manejo de las infecciones activas en las que participan bacterias anaerobias, protozoarios, nematodos o algunos virus. ${ }^{6,7}$

\section{FARMACOCINÉTICA}

Después de la administración oral de nitazoxanida, es completamente biotransformado por hidrólisis a su metabolito activo tizoxanida (desacetilnitazoxanida). Se reporta una vida media de seis minutos para nitazoxanida y no se detectan concentraciones plasmáticas del medicamento. La tizoxanida es el metabolito con actividad antiviral que alcanza concentraciones plasmáticas máximas entre uno a 
cuatro horas después de una dosis oral. La biodisponibilidad de la suspensión oral en comparación con la tableta es de $70 \%$. Su administración con alimentos incrementa la concentración plasmática máxima entre un 10 a $50 \%$ y modifica el tiempo máximo en una hora. ${ }^{8}$ El Alinia BioPharma reporta un incremento del $2 \%$ en la concentración plasmática máxima con la suspensión oral de nitazoxanida cuando se administra con alimentos $(5.37 \pm 1.92$ vs $5.49 \pm 2.06 \mu \mathrm{g} / \mathrm{mL}$ ) y una disminución de una hora en la $T_{\text {max }}$ (mediana de 1.5 vs 2.5 horas) y un aumento de $45 \%$ en área bajo la curva con alimentos $(21.3 \pm$ 10.5 vs $30.2 \pm 12.3 \mu \mathrm{g}-\mathrm{h} / \mathrm{mL}) .^{9}$

El metabolito activo se une ampliamente a proteínas plasmáticas (99.9\%). La tizoxanida se elimina en dos terceras partes de la dosis por heces, y un tercio por vía urinaria. La tizoxanida sufre conjugación con ácido glucurónido, que es eliminado por orina y bilis. La nitazoxanida tiene una vida media de uno a 1.6 horas y su metabolito inactivo (glucurónido de tizoxanida) tiene una vida media de siete horas. ${ }^{8,9}$

\section{NITAZOXANIDA Y EMBARAZO}

La nitazoxanida se clasifica de acuerdo con la FDA en el grupo de medicamentos clase $B_{M}$, es decir, estudios en animales preñados no han mostrado riesgo fetal y no existen estudios controlados en animales que confirmen teratogenicidad. Investigaciones en ratas a dosis altas (48 veces mayor a la dosis terapéutica) y en conejos (tres veces mayor) no producen daño fetal. Se desconoce si el metabolito activo de nitazoxanida (tizoxanida) atraviesa la barrera placentaria; su peso molecular bajo puede favorecer su paso por la placenta, pero su alta unión a proteínas plasmáticas (99.9\%) limita su distribución hacia el feto o embrión.

No existen estudios controlados en mujeres en el primer trimestre del embarazo que reporten reacciones adversas en el feto, ni hay evidencia de riesgo en el segundo o tercer trimestre del embarazo. Ante la falta de experiencia clínica en seres humanos, no se recomienda su empleo en mujeres embarazadas. ${ }^{10}$

\section{LACTANCIA}

Una dosis de $500 \mathrm{mg}$ de nitazoxanida en mujeres en lactancia produce bajas concentraciones de su metabolito activo tizoxanida en leche materna; Hadad GM y colaboradores evaluaron en una mujer de 30 años en periodo de lactancia la concentración de tizoxa- nida en leche materna después de la administración de $500 \mathrm{mg}$ de nitazoxanida por vía oral, obteniendo una concentración de $1.4 \mathrm{mg} / \mathrm{mL}$ después de seis horas. ${ }^{11}$

\section{NITAZOXANIDA EN INFECCIONES VIRALES}

La nitazoxanida suprime la replicación viral y activa proteínas intracelulares antivirales. ${ }^{6}$ La nitazoxanida y su metabolito activo circulante tizoxanida inhiben la replicación de un amplio rango de virus tanto RNA como DNA. Tiene acción contra 16 cepas de influenza A/H1N1, H3N2, H3N2v, H3N8, H5N9, H7N1 y una cepa de influenza $B$.

Actúa en contra de virus sincitial respiratorio, norovirus, dengue, fiebre amarilla, virus de la encefalitis japonesa, rotavirus, hepatitis $\mathrm{B}$ y $\mathrm{C}$, incluso contra el virus de la inmunodeficiencia humana, coronavirus del SARS y MERS, por lo cual se le considera como un fármaco con amplio espectro antiviral.

En patología respiratoria, la nitazoxanida ha demostrado una actividad antiviral importante contra virus respiratorios tales como los de la familia Orthomyxoviridae (género influenza, especies A y $B)^{12}$ y más recientemente contra representantes de la familia Paramyxoviridae (principalmente contra las especies de parainfluenza). ${ }^{12,13}$

Esto se observó en el estudio de Haffizulla y su equipo de trabajo, donde participaron 624 pacientes de entre 12 y 65 años, atendidos en 74 distintos centros estadounidenses debido a cefalea y sintomatología compatible con influenza en las 48 horas previas al inicio del protocolo. ${ }^{12}$

Tras fijar como objetivo primario el tiempo transcurrido desde la primera dosis y el alivio de la sintomatología, estos pacientes fueron distribuidos al azar (en proporción 1:1:1) para recibir dos veces al día durante cinco días nitazoxanida (300 o 600 $\mathrm{mg}$ ) o placebo; al tratamiento, siguió un periodo de monitoreo durante 28 días. ${ }^{12}$

Ambas dosis de nitazoxanida (sobre todo la de 600 $\mathrm{mg}$, dos veces al día) fueron eficaces para reducir el tiempo de duración de la sintomatología: 95.5 horas con $600 \mathrm{mg}$ de nitazoxanida (intervalo de confianza $\left[\mathrm{IC}_{95}\right]: 84.0$ a $108.0 ; p=0.0084$ respecto de placebo) y 109.1 horas con $300 \mathrm{mg}$ de nitazoxanida $\left(\mathrm{IC}_{95}: 96.1\right.$ a $129.5 ; p=0.52$ respecto de placebo) vs 116.7 horas con placebo $\left(\mathrm{IC}_{95}: 108.1\right.$ a $\left.122.1 ; \mathrm{p}=0.52\right) .{ }^{12}$

Las prevalencias de reacciones adversas fueron semejantes en los tres grupos, con predominio de 
Rev Latin Infect Pediatr. 2021; 34 (2): 94-99

cefalea $(6 \%$ con $300 \mathrm{mg}$ de nitazoxanida vs $8 \%$ con $600 \mathrm{mg}$ nitazoxanida vs $11 \%$ con placebo) y de diarrea $(2 \%$ con $300 \mathrm{mg}$ de nitazoxanida vs $3 \%$ con placebo vs $8 \%$ con $600 \mathrm{mg}$ de nitazoxanida). ${ }^{12}$

La conclusión es que nitazoxanida (300 o 600 $\mathrm{mg}$, dos veces al día) vs placebo es eficaz para disminuir el tiempo de duración de la sintomatología: 95.5 horas con $600 \mathrm{mg}$ de nitazoxanida, 109.1 horas con $300 \mathrm{mg}$ de nitazoxanida y 116.7 horas con placebo. ${ }^{12}$

La publicación de Piacentini y colaboradores se enfocó en los efectos de nitazoxanida y los de tizoxanida sobre los mecanismos de membrana que confieren una alta infectividad a los virus de la familia Paramyxoviridae (formados por proteínas de unión al receptor y por la proteína de fusión F). ${ }^{13}$

La nitazoxanida y su metabolito actuaron como inhibidores no-competitivos del sitio ERp57, lo cual produjo interrupciones en la señalización de la proteína $\mathrm{F}$ hacia la membrana plasmática del huésped, obstaculizando en esta forma la infección; a partir de estos efectos, la nitazoxanida se reveló nuevamente como una molécula viable para el combate contra los virus respiratorios que producen enfermedad en humanos. ${ }^{13}$

La nitazoxanida y su metabolito activo interrumpen la señalización de la proteína $F$ hacia la membrana plasmática del huésped; en esa forma, obstaculiza la infección por Paramyxoviridae. ${ }^{13}$

En gastroenteritis, una revisión sistemática de 14 estudios realizada por Tan y su equipo (cinco estudios controlados y aleatorizados, tres estudios de cohortes y seis reportes de casos) donde se evaluaron los efectos de nitazoxanida en pacientes pediátricos y adultos, con o sin inmunocompromiso, que exhibían sintomatología de gastroenteritis producida por adenovirus, norovirus o rotavirus ${ }^{14}$ mostró la eficacia de nitazoxanida para disminuir la duración de la enfermedad activa (entre 23 y 54 días con nitazoxanida vs entre 60 y 103.5 días con placebo; $\mathrm{p}<0.02$ para todas las comparaciones), reducir el tiempo de hospitalización (entre 68 y 81 horas con nitazoxanida vs entre 90 y 108 horas con placebo) y controlar la sintomatología de diarrea, náuseas y dolor abdominal. ${ }^{14}$

Si bien $22 \%$ de los participantes experimentó decoloración de la orina, porcentajes muy pequeños desarrollaron como reacción adversa (leve y autolimitado) dolor abdominal (4\%) o cefalea (2\%). ${ }^{14}$

Nitazoxanida vs placebo es eficaz para disminuir la duración de la enfermedad activa (entre 23 y 54 días con nitazoxanida vs entre 60 y 103.5 días con placebo) y reducir el tiempo de hospitalización (entre 68 y 81 horas con nitazoxanida vs entre 90 y 108 horas con placebo). ${ }^{14}$

En hepatitis, la nitazoxanida ha sido evaluada en pacientes con infección por VHB (500 mg, dos veces al día) y se ha observado que reduce tempranamente los marcadores de infección en 22 a 33\% de pacientes, y cuenta con eficacia sostenida en el mediano plazo en $89 \%$ de ellos. ${ }^{15}$

En pacientes con infección por VHC, la nitazoxanida (500 mg, dos veces al día) reduce los marcadores de infección en hasta $30 \%$ de pacientes. En estos casos, el tratamiento triple con nitazoxanida (500 mg, dos veces al día) + peginterferón alfa de tipo 2-a (180 $\mu \mathrm{g}$ cada semana) más ribavirina (1,000 a 1,200 mg, una vez al día) cuenta con eficacia mayor para reducir tempranamente los marcadores de infección y producir un control sostenido, respecto al manejo convencional con peginterferón alfa de tipo 2-a más ribavirina, y también al tratamiento dual con nitazoxanida más peginterferón alfa de tipo $2-a .{ }^{16}$

\section{EFICACIA EN COVID-19}

Haffizulla y colaboradores demostraron que la administración de $600 \mathrm{mg}$ cada 12 horas por cinco días disminuye la duración de los síntomas en casos con influenza aguda no complicada, con pocas reacciones adversas. ${ }^{12}$ En un estudio donde se compara la concentración plasmática máxima y la concentración en el órgano blanco (índice Cmax/EC50), se identificó que la tizoxanida alcanza concentraciones inhibitorias efectivas para producir su efecto antiviral en los diferentes órganos. ${ }^{17}$

Nitazoxanida muestra una excelente actividad in vitro contra SARS-CoV-2, con una concentración inhibitoria IC50 de $0.68 \mu \mathrm{g} / \mathrm{mL}$ (2.2 uM) y un IC90 de $1.84 \mu \mathrm{g} / \mathrm{mL}$ (5.6 uM). ${ }^{18,19}$ La dosis de $500 \mathrm{mg}$ cada seis horas de nitazoxanida con alimentos permite alcanzar concentraciones terapéuticas en los pacientes enfermos. Cuando se utilizan intervalos de administración, como cada ocho o 12 horas, se observan concentraciones plasmáticas por debajo de la concentración efectiva mínima. ${ }^{12}$

La actividad antiviral de la nitazoxanida se debe en parte a la amplificación de la respuesta antiviral del interferón e incrementa la RIG-1 (sensor RNA citoplasmático), GADD34 (proteína induce daño DNA y detiene crecimiento), PKR (proteíncinasa 
R) y efectores MAVS, interferones y múltiples ISGs (genes estimulador interferón). Además, la nitazoxanida tiene la capacidad de inhibir la producción de citocinas proinflamatorias, incluyendo TNF- $\alpha$, interleucinas IL2, IL4, IL5, IL6, IL8 y IL10 en células mononucleares. ${ }^{18-20}$

Aun cuando el mecanismo de acción de nitazoxanida contra SARS-CoV-2 es desconocido, se ha reportado que interfiere con la $\mathrm{N}$-glicosilación de hemaglutinina en el virus de la influenza. Por otra parte, potencia la producción de interferón tipo 1 y favorece la broncodilatación de las vías aéreas, por inhibición de la TMEM16A en los canales de cloro activados por calcio, hallazgo que puede contribuir a aliviar los síntomas asociados a COVID-19. ${ }^{19,21}$

\section{SEGURIDAD Y TOLERABILIDAD}

En forma dependiente de la dosis, la administración de nitazoxanida se relaciona con una baja prevalencia de efectos secundarios tales como cefalea ( 6 a $8 \%$ de pacientes) y diarrea ( 2 a $8 \%$ de pacientes). ${ }^{12}$ Aun cuando $22 \%$ de los pacientes tratados con nitazoxanida muestra decoloración de la orina, sólo $4 \%$ desarrolla dolor abdominal y $2 \%$ cefalea; en estos dos últimos casos, los episodios son leves y de naturaleza autolimitada. ${ }^{14}$

Tanto en pacientes con infección por VHB como por VHC, la administración de nitazoxanida induce tasas muy bajas de episodios (leves, transitorios y autolimitados), semejantes a las tasas asociadas con placebo, de diarrea o dolor epigástrico.

En una revisión de estudios clínicos de fase 2 y 3 de farmacología clínica con nitazoxanida, en bases de datos (EMBASE y MEDLINE) se encontraron nueve estudios controlados: cinco ensayos comparando nitazoxanida con placebo y cuatro contra antimicrobianos (metronidazol, vancomicina y terapia antituberculosa). Se identificó que no hay diferencias estadísticamente significativas entre nitazoxanida (44.2\%) en comparación con placebo o antimicrobiano (43.2\%), que sólo el $3.4 \%$ de las reacciones adversas fueron graves $(3.9 \%$ grupo control), siendo las reacciones gastrointestinales las más frecuentes (dolor abdominal) y se incrementaban conforme aumenta la dosis. Las pruebas de función hepática mostraron alteraciones en 21 pacientes de un total de $805 .{ }^{22}$

La nitazoxanida demostró un perfil de seguridad adecuado, muy similar a placebo a las dosis aprobadas. Hasta el 13 de junio de 2020, se han registrado 12 ensayos clínicos en la base ClinicalTrials.gov, cinco de ellos en fase de reclutamiento de pacientes. ${ }^{23}$

Hasta el momento, la única interacción medicamentosa con nitazoxanida documentada es con anticoagulantes cumarínicos; esta interacción eleva las concentraciones plasmáticas de éstos y prolonga el tiempo de protrombina. ${ }^{7}$

\section{EVIDENCIAS RECIENTES EN COVID-19}

Hace unos días, el Ministerio de Ciencia de Brasil dio a conocer de manera general los resultados obtenidos por la Dra. Patricia Rieken Macedo Rocco y colaboradores de la Universidad Federal de Río de Janeiro, en los que observó con el uso de nitazoxanida en 1,572 voluntarios disminución de carga viral de SAR-CoV-2 y mejoría de manifestaciones clínicas en casos de COVID-19 leves. ${ }^{24}$

J Meneses Calderón y su equipo recientemente publicaron un artículo, en el cual nos da a conocer su experiencia y concluye que la nitazoxanida puede utilizarse en el tratamiento de pacientes con COVID-19 con resultados exitosos..$^{25}$ Y Marcelo Silva presenta un trabajo donde demuestra en pacientes reducción de carga viral $\geq 35 \%$ desde el inicio hasta el día siete de tratamiento significativamente mayor para nitazoxanida en comparación con el placebo (47.8 frente a $15.4 \%$; $\Delta 34.6 \%$; IC del $95 \%$ : 64.7 ; 4.6: $p=0.037) .{ }^{26}$

\section{REFERENCIAS}

1. Pizzorno A, Padey B, Terrier O, Rosa-Calatrava M. Drug repurposing approaches for the treatment of influenza viral infection: reviving old drugs to fight against a long-lived enemy. Front Immunol. 2019; 10: 531. doi: 10.3389/fimmu.2019.00531.

2. Rossignol JF. Nitazoxanide: a first-in-class broad-spectrum antiviral agent. Antiviral Res. 2014; 110: 94-103.

3. Jasenosky LD, Cadena C, Mire CE et al. The FDA-approved oral drug nitazoxanide amplifies host antiviral responses and inhibits Ebola virus. iScience. 2019; 19: 1279-1290.

4. Rossignol JF. Nitazoxanide, a new drug candidate for the treatment of Middle East respiratory syndrome coronavirus. J Infect Public Health. 2016; 9 (3): 227-230.

5. Trabattoni D, Gnudi F, Ibba SV et al. Thiazolides elicit antiviral innate immunity and reduce HIV replication. Sci Rep. 2016; 6: 27148. doi: 10.1038/srep27148.

6. University of Alberta. Departments of Computing Science \& Biological Sciences; The Metabolomics Innovation Centre, DrugBank. Nitazoxanide. [Consultado 5 de febrero de 2020] Disponible en: www.drugbank.ca/drugs/DB00507

7. PR Vademecum. Nitazoxanida. [Consultado 21 de marzo de 2020] Disponible en: https://mx.prvademecum.com/ principio-activo/nitazoxanida-2760// 
Rev Latin Infect Pediatr. 2021; 34 (2): 94-99

8. Welzel DM, Philips MA. Chemotherapy of protozoal infection amebiasis, giardiasis, trichomoniasis, trypanosomiasis, leishmaniasis and other protozoal infection. In: Goodman LS, Brunton LL, Chabner B, Knollmann BC. Goodman \&Gilman. The pharmaceutical basis of therapeutics. 13 ed. New York, USA: McGraw Hill Education; 2018. pp. 987-99.

9. Nitazoxanida tablets. Clinical pharmaceutical and biopharmaceutical reviews, No 21-497, 2004. [Consultado 28 de abril de 2020] Disponible en: https://www.accessdata.fda. gov/drugsatfda_docs/nda/2004/21-497_Alinia_BioPharmr.pdf

10. Briggs GG, Freeman RK, Yaffe SJ. Drugs in pregnancy and lactation. A reference guide to fetal and neonatal risk. 8th ed. Philadelphia: Lippincott Williams \& Wilkins; 2008. pp. 1308-1309.

11. Hadad GM, Abdel-Salam RA, Emara S. Validated and optimized high performance liquid chromatographic determination of tizoxanide, the main active metabolite of nitazoxanide in human urine, plasma and breast milk. J Chromatogr Sci. 2012; 50: 509-515.

12. Haffizulla J, Hartman A, Hoppers M et al. Effect of nitazoxanide in adults and adolescents with acute uncomplicated influenza: a double-blind, randomised, placebo-controlled, phase 2b/3 trial. Lancet Infect Dis. 2014; 14 (7): 609-618.

13. Piacentini S, La Frazia S, Riccio A et al. Nitazoxanide inhibits paramyxovirus replication by targeting the Fusion protein folding: role of glycoprotein-specific thiol oxidoreductase ERp57. Sci Rep. 2018; 8 (1): 10425. doi: 10.1038/s41598018-28172-9.

14. Tan E, Cawcutt K, Zomok C et al. Activity of nitazoxanide against viral gastroenteritis: a systematic review. Int J Travel Med Glob Health. 2017; 5 (4): 107-112.

15. Rossignol JF, Bréchot $C$. A pilot clinical trial of nitazoxanide in the treatment of chronic hepatitis B. Hepatol Commun. 2019; 3 (6): 744-747.

16. Keeffe E, Rossignol JF. Treatment of chronic viral hepatitis with nitazoxanide and second generation thiazolides. World J Gastroenterol. 2009; 15 (15): 1805-1808.

17. Arshad $\mathrm{U}$, Pertinez $\mathrm{H}$, Box $\mathrm{H}$ et al. Prioritization of antiSARS-Cov-2 drug repurposing opportunities based on plasma and target site concentrations derived from their established human pharmacokinetics. Clin Pharmacol Ther. 2020; 108 (4): 775-790. doi: 10.1002/cpt.1909.

18. Srivatsan Padmanabhan M, Tech KPM. Nitazoxanide-a potential ally in the treatment of COVID-19. 2020. doi: 10.13140/RG.2.2.22854.83527.

19. Mahmoud DB, Shitu Z, Mostafa A. Drug repurposing of nitazoxanide: can it be an effective therapy for COVID-19? J Genet Eng Biotechnol. 2020; 18 (1): 35.

20. Padmanabhan S. Potential dual therapeutic approach against SARS-CoV-2/COVID-19 with nitazoxanide and hydroxychloroquine. ResearchGate. 2020. doi: 10.13140/ RG.2.2.28124.74882.

21. Khatri M, Mago P. Nitazoxanide/Camostat combination for COVID-19: an unexplored potential therapy. Chem Biol Lett. 2020; 7 (3): 192-196.

22. Pepperrell T, Pilkington V, Owen A, Wang J, Hill AM. Review of safety and minimum pricing of nitazoxanide for potential treatment of COVID-19. J Virus Erad. 2020; 6 (2): 52-60.

23. Dos Santos WG. Natural history of COVID-19 and current knowledge on treatment therapeutic options. Biomed Pharmacother. 2020; 129: 110493.

24. Rocco PRM, Silva PL, Cruz FF et al. Early use of nitazoxanide in mild Covid-19 disease: randomised, placebo-controlled trial. Eur Respir J. 2021. doi: 10.1183/13993003.037252020.

25. Meneses Calderón J, Figueroa Flores MDR, Paniagua Coria L, Briones Garduño JC, Meneses Figueroa J, Vargas Contretas $\mathrm{MJ}$ et al. Nitazoxanide against COVID-19 in three explorative scenarios. J Infect Dev Ctries. 2020; 14 (9): 982-986.

26. Silva M, Espejo A, Pereyra M, Lynch M, Thompson M, Taconelli $\mathrm{H}$ et al. Efficacy of Nitazoxanide in reducing the viral load in COVID-19 patients. Randomized, placebo-controlled, single-blinded, parallel-group, pilot study. medRxiv. 2021. Available in: https://doi.org/10.1101/2021.03.03.21252509

Financiamiento: Ninguno.

Conflicto de intereses: Ninguno.

Correspondencia:

Rodrigo Romero Feregrino

E-mail: drrodrigo@idisalud.com 